\title{
Journal Citation Report 2020 and Impact Factor of Journal of Korean Neurosurgical Society
}

\author{
Hee-Jin Yang, ${ }^{1}$ Chul-Kee Park, ${ }^{2}$ Moonyoung Chung ${ }^{3}$ \\ Editor in Chief, Journal of Korean Neurosurgical Society; Department of Neurosurgery, Seoul National University Boramae Hospital, Seoul, \\ Korea \\ Associate Editor, ${ }^{2}$ Journal of Korean Neurosurgical Society; Department of Neurosurgery, Seoul National University Hospital, Seoul National \\ University College of Medicine, Seoul, Korea \\ Managing Editor, Journal of Korean Neurosurgical Society; Department of Neurosurgery, Soonchunhyang University Bucheon Hospital, \\ Soonchunhayng University College of Medicine, Bucheon, Korea
}

\section{BRIEF OVERVIEW OF THE JOURNAL CITATION REPORTS (JCR) $)^{1)}$}

Since the publication of Medicina Curiosa, the first English medical journal in 1684, there has been exponential growth in the numbers of medical journals. It is roughly estimated that there are about 30000 scientific journals in the world. In the circumstances of overflowing numbers of medical journals, progress of their own medical journal is one of the primary goals of many medical societies and their members. After the introduction of impact factor by Eugene Garfield, it has become a major standard for evaluating the quality of a journal ${ }^{2}$. That is why editors of many medical journals anticipate for the updated JCR to be released in end of the June every year.

\section{STATUS OF JOURNAL OF KOREAN NEURO- SURGICAL SOCIETY (JKNS) IN 2020 JCR}

The 2020 impact factor (IF) of the JKNS was 1.729 , which is a $26 \%$ increase from last year (1.376). This rise in IF means that it is maintaining the upward trend that has continued in recent years, which is very encouraging (Fig. 1). Since JKNS was registered in the Scientific Citation Index in 2008 and received the first IF of 0.155 from 2009 JCR, there has been relatively steady state of IF around 0.6 to 0.7 for several years. It is in recent years that IF has started to increase remarkably, and this is the result of the unremitting efforts of all the members of the Korean Neurosurgical Society (KNS). Awareness of the importance of journal and support from KNS were also of great help ${ }^{3,4)}$.

Total citation number of articles published in JKNS was 294 during this period. The percentile of journal ranking of $J K N S$ according to the IF was $27.12 \%$, and $15.14 \%$ in the surgery category, and the clinical neurology category, respectively. This ranking is comparable to those of last year, indicating that the scientific influence of JKNS is currently stationary (Fig. 1).

Looking at the number of citations of the top-cited articles, there are one paper with 12 citation and two papers with $11 \mathrm{ci}-$ tations. A total of 11 papers have been cited more than six

- Received : August 18, 2021 • Accepted: August 23, 2021

- Address for reprints : Hee-Jin Yang

Department of Neurosurgery, Seoul National University Boramae Hospital, 20 Boramae-ro 5-gil, Dongjak-gu, Seoul 07061, Korea Tel : +82-2-870-2303, Fax : +82-2-870-3863, E-mail : nsyangdr@gmail.com, ORCID : https://orcid.org/0000-0002-6413-1363

This is an Open Access article distributed under the terms of the Creative Commons Attribution Non-Commercial License (http://creativecommons.org/licenses/by-nc/4.0) which permits unrestricted non-commercial use, distribution, and reproduction in any medium, provided the original work is properly cited. 


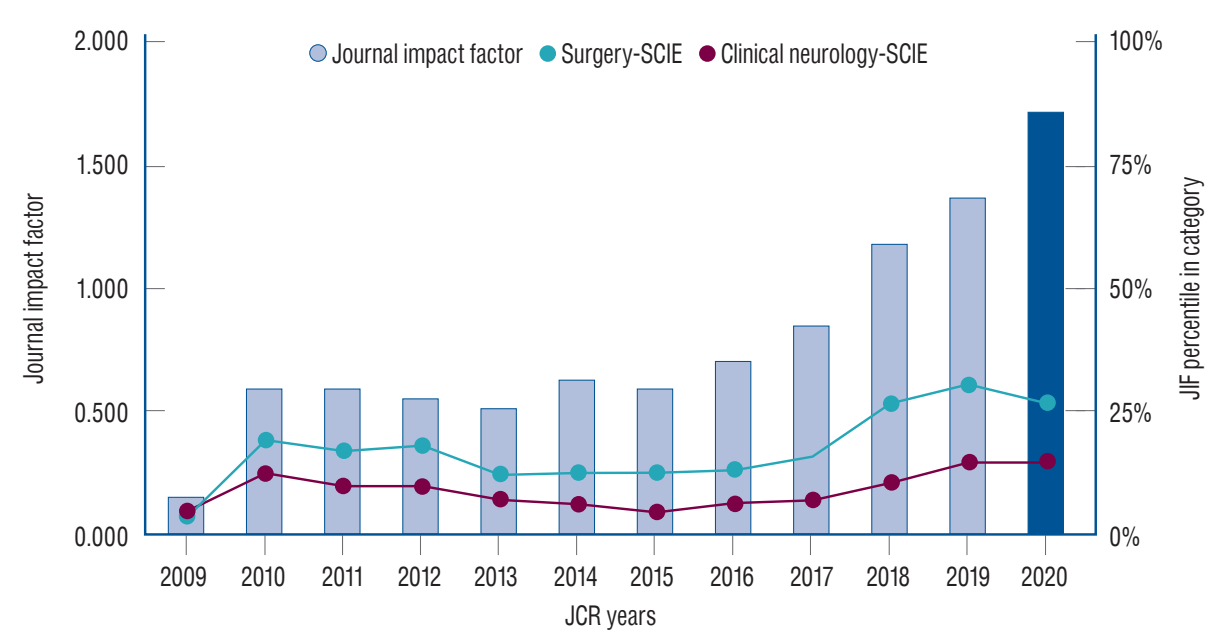

Fig. 1. Trends in years of the journal impact factor (JIF) and its percentile rank in category of the Journal of Korean Neurosurgical Society. Adapted from the 2020 Journal Citation Reports1). SCIE : science citation index expanded, JCR : journal citation reports.

times, of which eight papers are review articles. One of the points worth mentioning is that the JKNS has low self-citation rate $(6.8 \%)$ which means the IF is thanks to citations mostly from other journals. The weak point is that there are still considerable percentage of articles without citation (32.9\%, 56 out of 170 articles), and the large portion of the articles cited only once (30.6\%, 52 out of 170 articles).

The recent development of JKNS is paid off from combination of efforts of the KNS members, authors and editorial board of the JKNS. At the same time, it is noteworthy that these continuous efforts are essential components not only for past reputation but also for future development of the JKNS. This is why we need to put in more effort than ever before for the development of JKNS. As part of such an effort, it is thought that giving priority to JKNS when members of KNS submit their manuscripts would be of great help to the development of JKNS.

\section{CONFLICTS OF INTEREST}

No potential conflict of interest relevant to this article was reported.

\section{AUTHOR CONTRIBUTIONS}

\section{Conceptualization : HJY, CKP, MC}

\author{
Data curation : MC \\ Formal analysis : CKP \\ Funding acquisition : $\mathrm{MC}$ \\ Methodology : HJY \\ Project administration : HJY \\ Visualization : HJY \\ Writing - original draft : HJY, CKP, CM \\ Writing - review \& editing : HJY, CKP, CM
}

\section{ORCID}

Hee-Jin Yang https://orcid.org/0000-0002-6413-1363

Chul-Kee Park https://orcid.org/0000-0002-2350-9876

Moonyoung Chung https://orcid.org/0000-0003-4733-6321

\section{References}

1. Clarivate Analytics : $\mathbf{2 0 2 0}$ journal impact factor, journal citation reports. Available at : https://jcr.clarivate.com/jcr/home

2. Garfield $\mathrm{E}$ : Citation indexes for science; a new dimension in documentation through association of idea. Science 122 : 108-111, 1955

3. Im SB, Chung M, Kim BT : The influential articles and title words in the Journal of Korean Neurological Society publications between the years 2016 to 2018. J Korean Neurosurg Soc 63 : 1-3, 2020

4. Yang HJ : Journal of Korean Neurosurgical Society from 2001 to 2006, the pivotal period of transition and preparation for making great stride.

J Korean Neurosurg Soc 62 : 131-135, 2019 